\title{
Performance of WiMAX Physical layer MIMO-OFDM System under Rayleigh Fading Channel
}

\author{
Rajidi Sahithi, V. Madhu Kumar, T.V.Ramana
}

\begin{abstract}
WiMAX infrastructure will meet the needs of multiple consumers at very low cost and without the time required to install a wired network, for those who need quick access to rural areas where cable systems cannot be feasible because of the distances and costs involved. Multi-Input (MIMO) technology in combination with orthogonal frequency-division multiplexing (MIMO-OFDM) is an appealing architecture for wireless systems of the next decade. This system level approach has been used in many WiMAX simulator developments, both public and private. In this paper we are investigating the performance of Wi-MAX PHY layer for PHY-layer modifications of $2 \times 2$ MIMO (multiple-input multiple-output), spatial multiplexing, to provide high throughput, bandwidth efficiency and low bit error rate at high data rates. The paper provides necessary description for the simulation process of WiMAX model, addresses the important elements for specific inaccuracies that are existed in the physical layer with the BER for various modulation schemes.
\end{abstract}

\section{Keywords : WiMAX; IEEE 802.16; OFDM; MIMO}

\section{INTRODUCTION}

The Broadband Wireless Access (BWA) is an innovative last-mile access infrastructure platform that provides high-speed internet connectivity both in urban and small-to medium-sized businesses in recent days. The Worldwide interoperability of microwave connectivity (WiMAX) platform of IEEE 802.16[1] for the BWA and its related technology group aims to offer high data rates to many customers in larger areas where broadband is not accessible. This is the first standard for fixed wireless access with a significantly higher bandwidth than many cellular networks across the industry [2]. Wireless broadband systems which have been around for several years, but the growth of this standard allows economical size that reduces equipment cost, ensures interoperability and reduces the operators investment risk. The data is split into several parallel sub carriers in an OFDM system at a reduced data rate and each is modulated and transmitted on a separate orthogonal subcarrier. It improves system long life and robustness of the process. Multiplexing of user data sources relies majorly on the operating frequency that is most suitable for connections completes OFDM. There is need of implementing the system that deals with signal processing for current day wireless applications. To make it possible with less resources, the integration of MIMO to OFDM is the solution. It is evident

\section{Revised Manuscript Received on December 13, 2019.}

Mrs.Rajidi Sahithi, Assistant Professor, Dept. of ECE, CMR College of Engineering \& Technolgy, Hyderabad, Telangana, India. Email: sahithiramu@gmail.com

Mr.V.Madhu Kumar, Research Scholar/ Assistant Professor, Dept. of ECE, Gitam Deeemed to be University/ JITS, Vishakapatnam/ Warangal, Telangana, India. Email: madhuvjits@gmail.com

Dr. T.V.Ramana, Associate Professor, Department of ECE,GITAM Deemed to be University, Vishakapatnam, India. Email: teppala@gmail.com that the difference for both IEEE 802.16 standards and WiMAX is important to understand in WiMAX applications to maintain the complexity less, error free communication, high frequency operation, and high-end applications in wireless applications as its overview is shown in Fig.1.

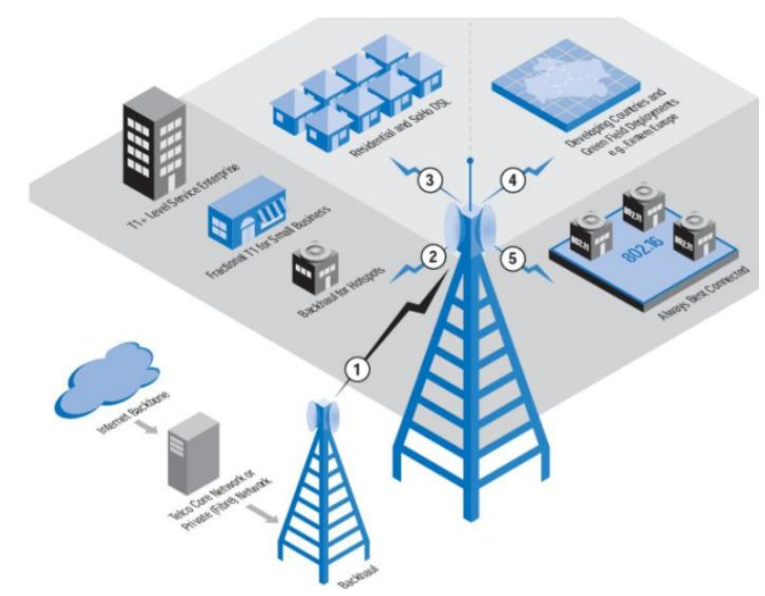

Fig. 1.Overview of Application scenarios [3]

WiMAX Forum with its support to multiple applications with different operating frequency modes makes this possible and these modes at this level are of greater conceptual importance as illustrated in Fig.2. The introducing of the OFDM with WiMAX framework brought about tremendous changes for business, industry, science and engineering can obtain very diverse outcomes the present day needs.

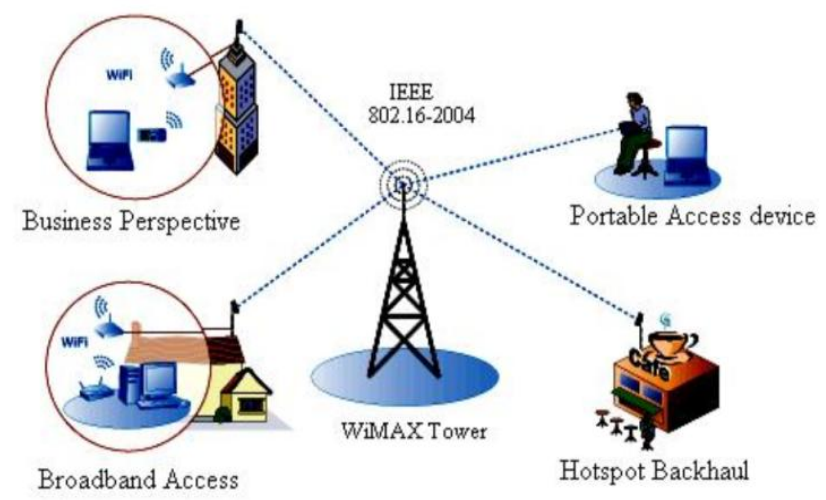

Fig. 2. WiMAX Scenario

However, new expertise posses with the characteristics without having the information known and that can be wrongly designed. Experts consequently must deliberate different simulation approaches to select the best one. Space-time coding is a technique for the coding of multiple antennas.

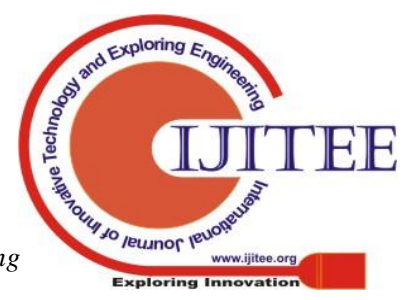


Coding is conducted in both the spatial and temporal realms in order to compare signals from various antennas at different times. The spatial-time comparison is used to use the MIMO channel decay and to eliminate propagation errors on the receiver. Space-time coding will gain flexibility and capacity improvement in bandwidth over spatially uncoded systems.

In this paper, basically work focus on how to model the OFDM framework with necessary changes in the physical layer of WiMAX. Then the effect of different modulation schemes over it is studied with the AWGN present in the channel.

\section{BACKGROUND WORKS}

Adaptive modulation's basic idea is to adapt different modulation techniques if a wireless communication system fails and changes in link. WiMAX takes full advantage of the coding and communication adjustment methodology. Another purpose of WiMAX is that it keep the modulation order higher/lower, when the channel is having bad effect on it in presence of noise. The amount of data transmitted per signal differs also with differences in modulation. For example, 64QAM will produce much higher output compared to QPSK. SNR should be suitable for the use of higher modulation to address distortion and interruption on the signal. In addition to $1 / 2$ rate convolutional error correction codes, lower data rates can be achieved through BPSK \& QPSK constellations. In this scenario, 1/2 means the system generates two one-bit communication codes. While all the existed modulation are depicted along with Shannon limit for various SNR values are illustrated in Fig.3that achieve higher data rates.

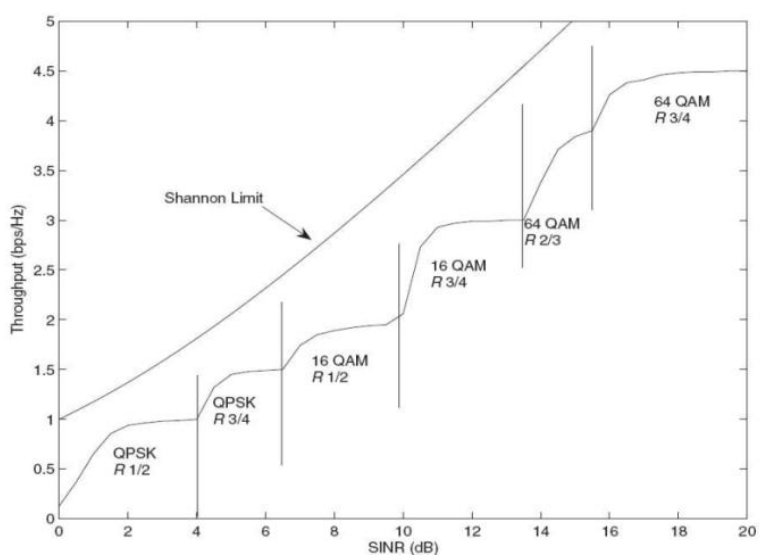

Fig. 3. Adaptive Modulation in WiMAX

In[4], the authors showed a method using Alamouti codes with linear MMSE detection techniques for the OFDM system. OFDM's orthogonal theory helps prevent inter-symbols conflict, while Alamouti offers signal protection to minimize errors particularly at a high signal-to-noise rate. To track the impact on our designed system, we have added White Gaussian Noise, which is also believed to have low correlation between the channels. In this study, the influence of high SNR on energy efficiency, i.e. energy consumption per bit, was analyzed using $2 \times 2$ MIMO architecture and a weak correlation coefficient.

In[5] the authors investigated the MIMO using a WiMAX (IEEE 802.16) space-time block code. WiMAX technology (Worldwide Interoperability for Microwave Access) capable of providing a high speed voice, image, video and data service, up to standard wireless MAN 802-16 base, is configured in the same way as a traditional mobile network. The WiMAX scope makes the system very appealing to consumers, but the BER at the low SNR is somewhat higher.

In[6], the authors illustrated the OFDM system's BER output with two separate equalizers ( $\mathrm{ZF}$ and MMSE). The proposed OFDM system is demonstrated with a range of BPSK, QPSK, 16-QAM or 64-QAM modulation technology using the AWGN (Additional White Gaussian Noise) and Rayleigh channels multi-path fading channels. In this article, an interleaved coded communication MIMO-OFDM system with different modulation schemes is suggested. A coded MIMO-OFDM system with 64 information subcarriers and a 16-QAM array is called. We also showed a system with 128 subcarriers. To simulate the OFDM system, the cyclic prefix length code is said to be the channel maximum delay that demonstrates channel capacity. MIMO-OFDM network improves performance, channel capacity and multi-user wireless communication's spectral efficiency.

The authors in [7] discuss WiMAX's summary of basic design and upgrade and WiMAX layout such as network and node structures, physical layer, and MAC level). WiMAX is a broad-based communication network. It support high bandwidth and provides an alternative to wired broadband like cable and DSL for hundreds of users per channel at speeds similar to those currently seen on the DSL, Cable or $\mathrm{T} 1$ connectivity.

\section{PROPOSED SYSTEM}

The operation begins with the generation of random bit stream, followed with the symbol formation, and later ceding of data is done.

Physical Layer Modeling:Digital modulation system is used as various modulation schemes are desirable at various data rates. The MIMO space-time diversity encoder is then used with multiple antennas. After this, to avoid the channel interferences suitable guard band is appended in between subcarriers, transformed to time domain. To resist against ICI cyclic prefix is added which is larger than the anticipated time spread. For Adaptive stream prediction methodology is applied the faded models of the transmitted signals are imposed on each antenna differently. Eventually, after demodulation and channel decoding, binary information data are collected.

\section{A. Channel Encoding}

It is evident that wireless channel with constantly changing relation, often with significant interruption. Channel coding, which has the main tasks of preventing and correcting wireless system transmission errors, for increasing the channel gain and reduces complexity in the receiver to obtain faster data rates for wireless applications. There are 3 stages in the 802. 16 channel coding chain: Randomizer, Forward Error Correction (FEC) and interleaving. 


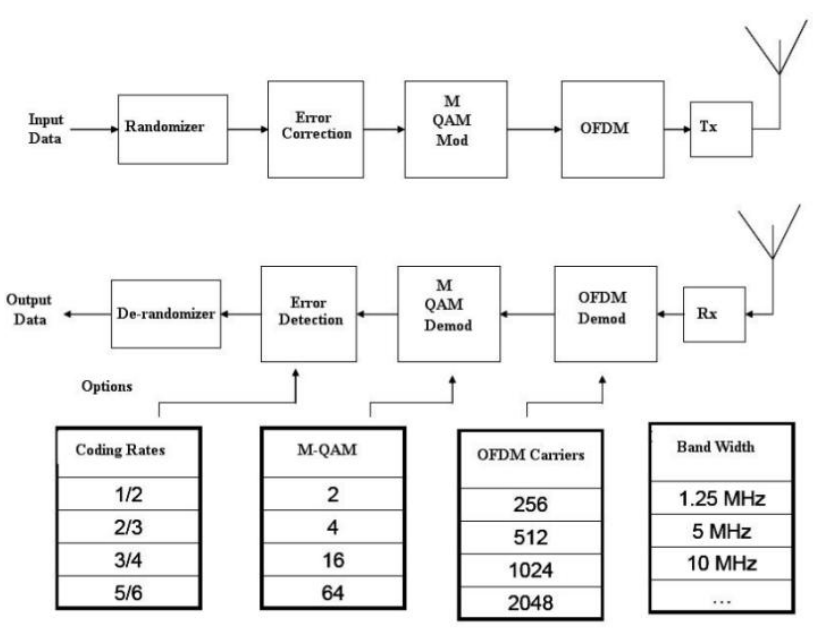

Fig. 4. Basic WiMAX Architecture

\section{Randomizer:}

Randomization is the first physical layer process following the receipt of the data packet from the MAC layer. On a bit by bit, Randomizer moves. Each burst is randomized in the transmitter and receiver. The scrambled information are meant to transform long 0 -or 1 -sequence sequences into short sequences to increase coding efficiency. A Pseudo Random Binary Sequence Generator, which is introduced using the Linear Feedback Shift Register, is the main component of randomization as shown in Fig.5.

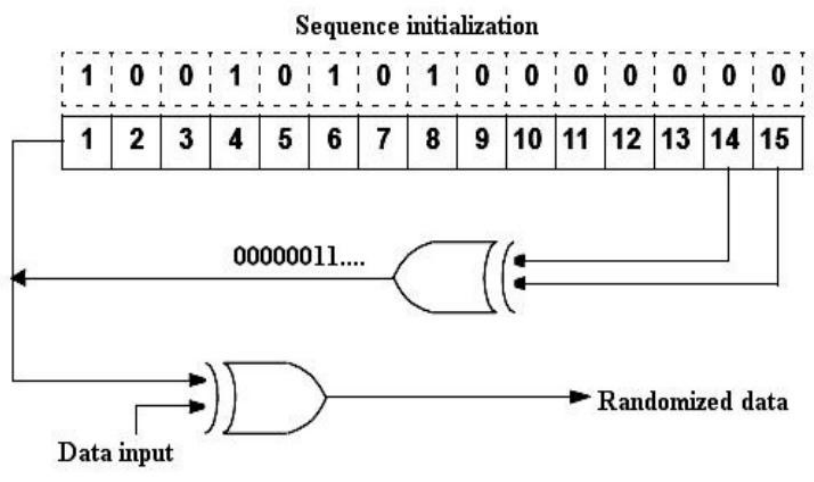

Fig. 5. PN Sequence Initialization

\section{Forward Error Correction:}

The code to fix errors is a crucial and significant method for the communication system design. This reduces power, delays, bandwidth and complexities of the device. The small improvements in error correction codes make sure suitable performance levels and channel gain which in turn overall quality involved for process. The contributions made by ECC strategies premised on multi-level modulation iterative coding to increase new possibilities for more cost-effective and robust design. The FEC is accomplished by using the Convolutional Codes and the Reed Solomon codes (correct burst errors at the byte level), which provide the additional coding benefit which measures the amount of additional SNRs required to deliver the same BER output in the same channel conditions in the case of uncoded message signal.

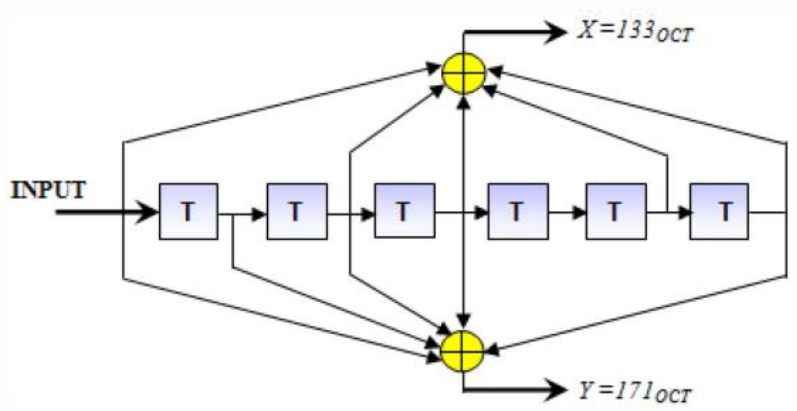

Fig.6. Convolutional Encoder $($ Rate $=1 / 2, \mathrm{~m}=7)$

\section{Interleaving:}

Interleaver is a tool that deterministically organizes the series of symbols. The size of the interleaver plays a key role in performance, thereby reducing the bit correlation. This paper selects the interleaver so that the information is written in column format and read in a row.

\section{B. Digital Modulation}

In this process data stream are initially undergo mapping operation and then applied for suitable modulation designated subcarriers after channel coding. We transferred the random values according to the specified constellation via adaptive modulation schemes. The data were modulated based on different modulation schemes such as BPSK, QPSK, and 16-QAM and 64-QAM modulation, depending upon their size. The reverse operation, defined as demodulation, is carried out by the recipient to retrieve the data transmitted.

\section{MIMO Encoder}

In this process the system comprises of 2 transmit and 2 receive antennas. This encoder requires a V-BLAST scheme used in this paper to further increase the spatial efficiency of $2 \times 2$ antennas.

\section{Spatial Multiplexing:}

Through this process, the data is supposed to sliced in to individual bit streams and broadcast on the same frequency channel from a separate transmitting antenna. If these signals come with sufficient and different spatial signatures on the receiver antenna array, they get separable to available as independent channels. The important concept associated with this process is to improve channel efficiency for higher values of SNR. It can be used with or without the knowledge of the channel that requires V-BLAST.

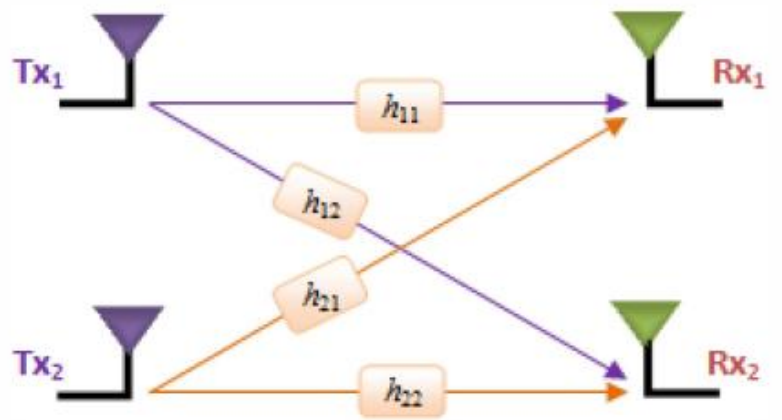

Fig. 7. MIMO Channel Model (2x2)

Consider the process of transmission consists of series of symbols $S_{1}, S_{2} \ldots \ldots . S_{\mathrm{n}}$. Then the grouped data are sent over 
2 antennas as shown in Fig.7. Send $S_{1}$ and $S_{2}$ during the first time slot and $S_{3}$ and $S_{4}$ during the second slot to avoid interference and maintain good orthogonality between symbols.Note that because we group and send two symbols in the only single slot that gives rise to saving the time and data gets doubled. For the $2 \times 2$ MIMO system with V-BLAST detector arranged in the matrix format as

$$
\left[\begin{array}{c}
r_{1} \\
r_{2}
\end{array}\right]=\left[\begin{array}{cc}
h_{i j} & h_{12} \\
h_{21} & h_{22}
\end{array}\right]\left[\begin{array}{c}
S_{1} \\
S_{2}
\end{array}\right]+\left[\begin{array}{c}
n_{1} \\
n_{2}
\end{array}\right] \text { (1) }
$$

Where, $r_{1}, r_{2}$ are treated to received symbol outputs, $h_{i j}$ is treated to channel matrix from $i^{\text {th }}$ to $j^{\text {th }}$ antennas, $S_{1}, S_{2}$ are treated to transmitted symbol inputs and $n_{1}, n_{2}$ are noise sources in both receive antennas.

\section{OFDM System}

The supposed multi-carrier system that divides provided channel bandwidth of the system into orthogonal channels, each with only a narrow range, and a separate sub-carrier. The system is reconfigured to resist against several channel impairments and independently operates irrespective of various effects of fading through the guard interval and cyclic prefix.

\section{Inverse Fast Fourier Transform (IFFT):}

In this operation every data block undergoes one of the modulation scheme and is followed by an S/P conversion, then new block transforms the data back to its sequential format at its performance. Therefore inverse FFT transforms back to the time domain of all the available subcarriers where the signal is predominantly in the time domain.

With the suitable IFFT block helpful in producing corresponding signal waveform frequency components that meets requirement of orthogonality as there is nothing in the subcarrier. FFT often translates the reverse process of IFFT that is needed for all the schemes.

\section{Cyclic Prefix Insertion:}

Subsequently carrying out Inverse Transform, each OFDM symbol has been added to the cyclic prefix (CP). It is seen that $\mathrm{CP}$ appears to be as replica for the OFDM conceptual data stream attached to it (see Fig. 8).

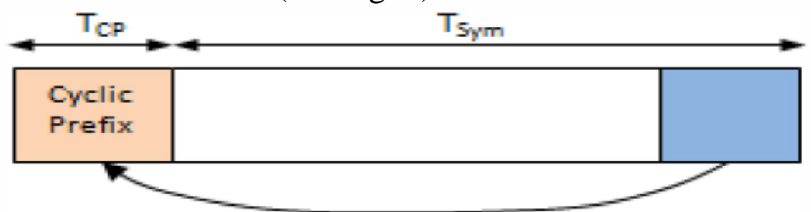

Fig. 8. Cyclic Prefix Insertion.

\section{E. Communication Channel}

The channels of communication are a form of communication medium between the transmitter and the receiver. We chose the Rayleigh channel design to simulate the interaction with Non Line of Sight (NLOS).

\section{RESULTS AND DISCUSSION}

In this section, initially modelling of WiMAX physical layer is done then considering some theoretical values for OFDM system is tabulated and also exhibit numerical results of the MIMO-OFDM system for different modulation systems are simulated on Matlab 2013a.

Table 1. Simulation parameters Parameter

Carrier Frequency Value

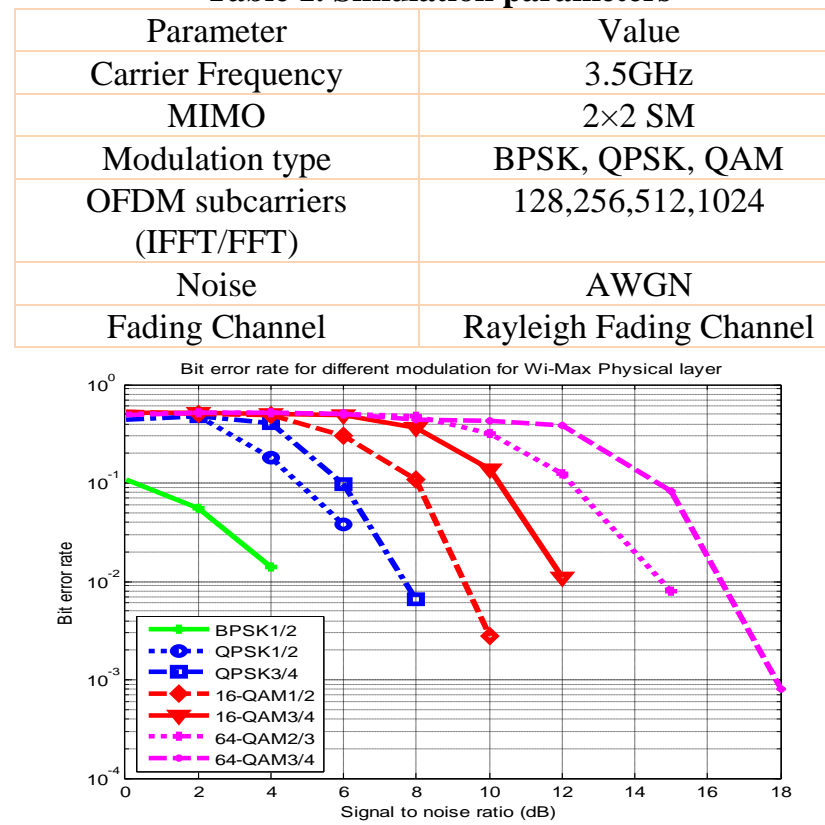

Fig.9. Bit Error Rate for Different Modulation for Wi-Max Physical Layer

\section{CONCLUSION}

In this paper, it is suggested a simulator in a physical layer on the flat Rayleigh fading channel for WiMAX-OFDM. The introduced PHY layer supports both modulation and coding schemes and the defined CP size. The BER is a primary indicator of the efficiency of a wireless communication network. In order to compare the performance of different modulation and coding systems, the BER curves were used.

\section{REFERENCES}

1. IEEE Standard for Local and Metropolitan area networks Part 16, The Institute of Electrical and Electronics Engineering, Inc. Std. IEEE 802.16d-2004.

2. Ghosh, A.; Wolter, D.R.; Andrews, J.G.; Chen, R., "Broadband wireless access with WiMax/802.16: current performance benchmarks and future potential", Communications Magazine, IEEE, Vol.43, Iss.2, Feb. 2005, Pages: 129- 136.

3. IEEE 802.16 and WiMAX, "http: //www.wimaxindustry.com /wp/papers/intel_80216_wimax.pdf', last accessed on 15.05.07.

4. Rohtang Saxena \& Neeraj Shrivastava (2016). "Energy Efficient System Using MIMO-OFDM" International Journal of Electrical, Electronics And Data Communication, Volume-4, Issue-12.

5. Mishra, Chandrakant \& Lal, Sonu, (2016). "Survey Paper on Performance Evaluation of WiMAX (IEEE 802.16) System using Space Time Block Coding Technique”, JARCSSE, 6(1), 682-686.

6. [6].Nidhi Kishor Molgi Suresh R. (2016) "Analysis ofMIMO-OFDM using different ModulationTechniques" International Journal of Computer Applications, Volume 153 - No 6

7. Seyedzadegan, Mojtaba \& Othman, Mohamed,(2013). "IEEE 802.16 WiMAX Overview,WiMAX Architecture”, International Journal ofComputer Theory and Engineering, 5(5), 784-787. 


\section{AUTHORS PROFILE}

Mrs. Rajidi Sahithi pursuing PhD in GITAM Deemed to be University, received M.Tech degree from JNTU Hyderabad in VLSI SYSTEM DESIGN in 2012. She is currently working as Assistant Professor in department of Electronics and Communication Engineering, CMR College of Engineering \& Technology, JNTU Hyderabad and has 10 years of teaching experience. Her research interest is Wireless Communications, published papers in various National and International Conferences and Journals.

Mr. V. Madhu Kumar, is a Research Scholar at Gitam Deemed to be University and currently working as Assistant Professor in JITS (Autonomous), Narsampet, Warangal. He has completed his Masters in DSCE from Jntu Hyderabad. He is the Member of IEEE and Life Member of ISTE. His research area is Wireless Communications and Signal Processing. His research papers are published in several International Journals.

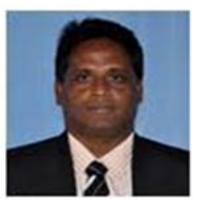

Dr. T. Venkata Ramana, as a diligent scholar has excelled in studies from his child hood. His dare to dream, to work smart and strive for nothing less than excellence and to enjoy the journey every step of the way made him to scale greater heights. He has obtained his Bachelor's Degree in Electronics \& Communications Engineering from Nagarjuna University. $\mathrm{He}$ has completed her Masters in Systems and Control Engineering from Osmania university. He has completed his Doctoral program Ph.D. in the area Satellite Communications from the Andhra University. He took up academic pursuit and served in an array of designations like Associate Professor \& Coordinator for M.Tech (Evening programs) of GITAM Deemed to be University. He also involved himself in teaching and research tasks. Above all he took up a mission to ignite the young minds so as to nurture the students with the potential to reach greater horizons. He strongly believed in himself, his mentors and peers and partners. 